\title{
Psychological Aspect of Rehabilitation and Return to Sport Following Lower Limb Injuries
}

\author{
Jan Marusic', Petra Dolenc ${ }^{1,2}$, Nejc Sarabon ${ }^{1,3,4}$ \\ Affiliations: 'University of Primorska, Faculty of Health Sciences, Izola, Slovenia, ${ }^{2}$ University of Primorska, Faculty of \\ Education, Koper, Slovenia, ${ }^{3}$ S2P, Science to Practice, Ltd., Laboratory for Motor Control and Motor Behaviour, Ljubljana, \\ Slovenia, ${ }^{4}$ University of Primorska, Andrej Marusic Institute, Izola, Slovenia
}

Correspondence: N. Sarabon, University of Primorska, Faculty of Health Sciences, Polje 42, SI-6310 Izola, Slovenia. E-mail: nejc.sarabon@fvz.upr.si

ABSTRACT In addition to an athlete's physical abilities, his or her mental state is also vital for optimal recovery and a successful return to sport after an injury. The purpose of the present review was to determine which psychological factors affect the success of rehabilitation and return to sport following the most frequent musculotendinous thigh injuries (hamstrings, hip adductors, knee extensors), and to present the main psychological screening tools and interventions that can aid the athlete's rehabilitation and return to sport. A systematic review of the literature did not reveal any relevant results related to those injuries; therefore, the search was expanded to whole lower limb injuries, which showed that several different psychological factors could have a significant impact on an athlete's rehabilitation and decision to return to sport. It can be presumed that these factors can similarly affect the post-injury process for thigh injuries, which should be considered when planning the rehabilitation programme. Systematically implementing psychological screening and psychological interventions (if needed) into the already established rehabilitation protocols would be reasonable for optimizing an athlete's recovery and for helping with the decision to return to sport.

KEY WORDS psychology, leg injuries, rehabilitation, return to sport

$@$ MJSSMontenegro

PSYCHOLOGY OF REHABILITATION AND RETURN TO SPORT

http://mjssm.me/?sekcija=article\&artid=197

\section{Introduction}

For those working in the field of sports injuries prevention and rehabilitation, knowing and understanding the factors that can affect the outcome of rehabilitation and the success of the athlete's return to sport after a sport injury is essential. Only by considering all the factors can an athlete optimally prepare (physically and mentally) for an effective return to the competition while minimizing the risks of re-injury. This is particularly important, since previous injury is one of the most critical risk factors (i.e., the most reliable predictor) for the occurrence of injury, especially amongst the most common musculotendinous injuries (Beijsterveldt, Port, Vereijken, \& Backx, 2012; Mosler et al., 2018).

There is a significant number of research and evidence-based protocols for successful rehabilitation after sports injuries in terms of the recovered functionality and physical performance of the injured body part/ joint. Nevertheless, even completely recovered physical abilities (achieving all the recommended milestones of the rehabilitation protocol) do not guarantee the athlete a successful return to sport. Ardern, Webster, Taylor, and Feller (2011) showed that there was a difference between the number of successful rehabilitations and the number of successful returns to sport. Part of that difference can be explained by various factors, such as injury in addition to the physical and functional limitations it causes, that can also affect the psychological state of an athlete. Furthermore, research shows several different psychological factors that can negatively affect the process of sport rehabilitation, its outcome and even the decision about continuing with sports career or not (Booth-Kewley et al., 2014; Kvist, Ek, Sporrstedt, \& Good, 2005). These facts raise the question of whether the current criteria for successful rehabilitation are appropriately defined, as they often focus only on the physical fitness of patients, while their mental state is, to a great extent, neglected.

Received: January 222020 | Accepted after revision: March 152020 | First published online: Septembar 012020

(c) 2020 by the author(s). License MSA, Podgorica, Montenegro. This article is an open access article distributed under the terms and conditions of the Creative Commons Attribution (CC BY).

Conflict of interest: None declared. 
Our review revealed a considerable lack of research about the psychological factors that can affect the successful outcome of rehabilitation and return to sport following the most common musculotendinous thigh injuries. These injuries represent a substantial problem in some sports, as they account for the majority of all sports injuries. Ekstrand, Hägglund, and Waldén (2011) showed that in soccer $92 \%$ of all injuries belong to the following muscle groups: $37 \%$ hamstrings, $23 \%$ hip adductors, $19 \%$ knee extensors, and $13 \%$ calf muscles. The impact of psychological factors on the recovery and return to sport after these injuries can only be deduced from a much more researched area of injuries: the knee joint, specifically the anterior cruciate ligament (ACL) injuries. Thus, the purposes of this paper are (based on a systematic literature review): (i) to verify which are the most important psychological factors that can affect the outcome of rehabilitation and the successful return to sport following lower limb sport injuries; (ii) to determine whether these psychological factors can similarly affect an athlete with a musculotendinous thigh injury during rehabilitation and the return to sport; (iii) to present the primary screening tools and interventions that can psychologically help an athlete and rehabilitation/prevention specialist. Identifying and monitoring psychological factors could help optimize preventative and rehabilitative programmes for these injuries and reduce the number of failed returns to sports. Therefore, an athlete's mental state after a sport injury should be just as carefully monitored and managed (if needed) as his/her physical abilities.

\section{Methods}

\section{Search strategy}

The original idea was to systematically review the literature about psychological factors that can affect athlete's rehabilitation or decision making about the return to sport specifically after the most frequent lower limb injuries in sports, such as American football, soccer and track and field, which are injuries to the hamstrings, knee extensors, and hip adductors. Given that initial search strings revealed no relevant results, suggesting a lack of research on this topic, we expanded the review to the whole lower limb injuries.

The PubMed database was searched in a systematic way for relevant articles published before October 2019. Search terms were divided into three concepts: Concept 1 included injury areas, Concept 2 included injury expressions, and Concept 3 included psychological aspects. Within each concept, the terms were grouped with the OR operator, and all three concepts were then combined with the AND operator into the following search string: (adductor OR hamstring OR groin OR rectus femoris OR quadriceps OR lower extremity OR ankle OR hip OR knee) AND (injury OR re-injury OR sport injury OR strain OR avulsion OR rupture) AND (psychology OR fear OR avoidance OR motivation OR anxiety OR psychological rehabilitation).

This search resulted in 2713 articles, which were then filtered by species (humans) and language (English), which resulted in 2340 articles. Each article was reviewed for inclusion with one of the following criteria: reports about the effect of psychological factors on rehabilitation outcome or return to sport after an injury of the lower extremity, reports about the psychological state of a patient/athlete after an injury of the lower extremity, reports about the impact of psychological interventions on rehabilitation outcome or patient's psychological state. Twenty-seven articles were eligible and thus included in this review.

\section{Results}

Psychological factors related to rehabilitation and return to sport after an ACL injury

Our review revealed a complete lack of research about the possible impact of psychological factors on an athlete's success of rehabilitation or return to sport after the injuries of hamstrings, knee extensors or hip adductors. In addition, the majority of the research about the psychological factors and their effects on rehabilitation and return to sport was done on patients/athletes with ACL tears (22 articles of 27 selected and included in this review). Most rehabilitation protocols after the reconstruction of the ACL (RACL) monitor patient's progress based on their injured knee ROM, degree of pain, overall knee functionality and strength of $\mathrm{m}$. quadriceps. Return to sport is recommended upon reaching $90 \%$ lateral symmetry in knee extension strength and one-legged hop test (Grindem, Snyder-Mackler, Moksnes, Engebretsen, \& Risberg, 2016). It turns out that even when these standards are accomplished, the successful return of the athlete to sport does not always happen. Ardern et al. (2011) concluded that as much as $90 \%$ of patients after the RACL regain normal knee functionality (measured as a single-limb hop for distance, isokinetic muscle strength or anterior knee laxity), but only half of the athletes return to the competitive sport. Psychological factors, such as fear of re-injury (Kvist et al., 2005; Lentz et al., 2015; Nwachukwu et al., 2019; Te Wierike, Van Der Sluis, Van Den Akker-Scheek, Elferink-Gemser, \& Visscher, 2013), anxiety (Kosy et al., 2019), self-efficacy (the individual's judgement about his/her ability to perform a particular task) (Baez, Hoch, \& Hoch, 2019; Beischer et al., 2019; Te Wierike et al., 2013) motivation (Sonesson, Kvist, Ardern, Österberg, \& Silbernagel, 2017), and increased rate of kinesiophobia (fear of movement) (Czuppon et al. 2014) may be the cause of such significant reduction of successful returns to sport after the RACL. Kinesiophobia and fear of re-injury are linked to reduced muscle endurance, muscle activation, dynamic knee stability, limited knee range of motion, self-reported function, and altered muscle recruitment strategies (Hsu, Meierbachtol, George, \& Chmielewski, 2017). Ardern, Taylor, Feller, Whitehead, and Webster (2013) and Te Wierike et al. (2013) reported a significant effect of internal health locus of control (the degree to which individuals perceive their ability to control life events). These authors assumed that the higher rate of patients' internal health locus of control enables better rehabilitation outcomes after the RACL, as it determines patients' self-esteem and their belief in themselves and 
in the positive outcome of rehabilitation, which could be a necessity for achieving good adherence to the rehabilitation programmes.

Everhart, Best, and Flanigan (2015) focused on predictive psychological factors for the recovery outcomes after RACL. They found that the patient's level of self-esteem, optimism, self-motivation, stress, social support, and self-identity as an athlete can predict the success of rehabilitation after RACL. They were able to connect these factors to the patient's level of pain and functionality of the injured knee, which can affect the successful return to sport. Furthermore, Masten et al. (2014) showed that the success of rehabilitation could be predicted from changes in certain psychosocial variables: a decrease in anxiety and an increase in susceptibility, self-efficacy and coping with pain. The decision to return to sport may also be influenced by the results of self-evaluation questionnaires on the functionality of the knee joint after the RACL. When using these, therapists must also consider the athlete's psychological state, which can negatively affect the results of the questionnaire. Patients diagnosed with severe depressive disorder reported significantly lower functionality after having equally successful RACL compared to psychologically healthy subjects (Garcia et al., 2016). Furthermore, athletes who did not return to sport had lower self-reported scores of the injured knee functionality after the RACL compared to the athletes who had a successful return to sport, even though both groups had similar (objectively measured) physical performance scores (star excursion balance test, single-legged hop tests, isokinetic knee flexors/extensors strength, step down endurance test) (Werner et al., 2018).

A qualitative study (Burland et al., 2018) with semi-structured interviews of 12 injured athletes revealed the following psychological factors (in addition to the fear of re-injury) that may influence the decision about returning to sports after the RACL: hesitation and lack of self-confidence to continue with a sport career, changes in (life) priorities, athletic identity, as well as expectations and assumptions about the rehabilitation process and its outcome. The participants had distorted beliefs about rehabilitation and were mentally unprepared, meaning that they did not know the proper psychological ways to manage the situation after the injury. Furthermore, the authors identified increased awareness of the injured knee as a psychological factor that can also have an adverse impact on the return to sport, since it is closely linked to the fear of re-injury and movement in general. This factor can be intensified when using various joint braces or kinesiotape (often used during rehabilitation), as it can also serve as a constant physical reminder of the injury and thus further increase the self-awareness of the affected joint (Burland et al., 2018). An additional factor that can affect the athlete's psychological state is the post-injury social support system (moral support and encouragement from teammates and coaches, and a good relationship with the therapist). It can have a significant positive or negative impact on rehabilitation outcome and return to sports. Burland et al. (2018) logically grouped the identified factors into the dynamic biopsychosocial model, developed by Wiese-Bjornstal, Smith, Shaffer, and Morrey (1998), which includes four main components:

- cognition (in addition to the athlete's post-injury thinking, it also includes various psychological factors that can influence the course and outcome of rehabilitation and the decision about returning to sport);

- affect (refers to the influence of emotions and different moods that surround an athlete after an injury);

- behaviour (consists of behaviours that involve the athlete's specific activities and efforts after an injury, which are highly influenced by cognition and affect);

- outcome (refers to the athlete's rehabilitation results and, most importantly, his/her decision to return to competition or to terminate his/her career).

These four components are interrelated and dynamic regarding the patient's response to the sport injury and rehabilitation process.

Psychological factors affecting rehabilitation of other lower limb injuries

Psychological factors have also been addressed and proven to be critical during the recovery from ankle injuries. Briet et al. (2016) reported a significant association between self-efficacy and the results of the Olerud-Molander Ankle Score questionnaire designed to assess the limitations associated with an ankle injury. Patients with lower levels of self-efficacy have been found to experience higher levels of pain and to have more physical limitations and more severe injury symptoms. In contrast, the severity of the injury or the degree of ankle sprain had no correlation with the results of the Score. Moreover, several studies have also confirmed a higher level of fear of re-injury and kinesiophobia, both in university athletes with a history of an ankle injury (Houston, Hoch, \& Hoch, 2017) and in athletes with chronic ankle instability (Houston, Van Lunen, \& Hoch, 2014).

Booth-Kewley et al. (2014) reported recovery expectations as the most significant predictive factor in the success of rehabilitation after musculoskeletal injury (knee, back or shoulder injuries) in soldiers. Individuals with high expectations were five times more likely to recover successfully than individuals with low expectations. The authors state different possible reasons for this: high expectations may be associated with less negative emotions about the injury and greater motivation for the patient to participate consistently in the rehabilitation process; it may also be related to the patient's physiological response, which can directly reduce pain and thus have a positive effect on the success of the recovery. In contrast, negative psychological factors, such as catastrophic thinking, appear to be strongly associated with greater pain intensity and disability after musculoskeletal trauma (Vranceanu et al., 2014). 


\section{Discussion}

Several psychological factors can affect an athlete's rehabilitation process and its outcome, as well as the success of returning to sports after a sport injury, particularly after RACL and ankle injuries. It can be assumed that the psychological factors affecting the return to sport after knee and ankle injuries could similarly affect the return to sport following the most frequent musculotendinous thigh injuries, the latter being the initial target of this review and the current and near-future research focus of its authors. These injuries can be divided according to severity into three grades. The first-grade injury is characterized by mild muscle strain, a minor decline in muscle strength, and required a few days of recovery. The second-grade injury is characterized by partial muscle tear, a greater decline in muscle strength and recovery is prolonged to several weeks. The third-grade injury is characterized by complete or near-complete tearing of muscle fibres, the recovery time can be extended up to six months, and surgery is required/preferred in most cases. Such thigh injuries are most comparable with the ACL injuries in terms of severity, need for surgery and time of absence from the competition. Given these similarities, the impact of psychological factors on the success of rehabilitation and return to sports could also be comparable. In the case of the first- or second-grade injuries, rehabilitation time is significantly shorter, which may also reduce the impact of psychological factors on return to sport. However, athletes with previous hamstring injuries have a high chance of injury recurrence, depending on the sport: $16 \%$ for soccer (Ekstrand, Hägglund, \& Waldén, 2011), 23\% for rugby (Brooks, Fuller, Kemp, \& Reddin, 2005) and Australian football (Orchard, Seward, \& Orchard, 2013), and $3 \%$ for American football (Feeley et al., 2008). Each re-injury could further impair the athlete's mental state and influence the decision about returning to the sport.

Given the repeatedly proven impact of different psychological factors on the athlete's recovery from an injury or return to sport, it would be reasonable to include additional measures in rehabilitation programmes. Consideration, assessment and monitoring of psychological factors often seem underestimated and underused in clinical practice, despite numerous studies indicating the significant impact of these factors on the successful return to sport. Merely monitoring the main psychological factors through a conversation or appropriate questionnaires would give a clearer picture of the athlete's psychological condition and help identify potential complications in returning to sport. Many established questionnaires can discover different psychological factors. To monitor the presence of kinesiophobia, the Tampa Scale questionnaire can be used (Miller, Kori, \& Todd, 1991). The Fear-Avoidance Beliefs Questionnaire is used to measure a patient's fear of pain and their avoidance of physical activity. The emotional response to pain can be evaluated by the Pain Catastrophizing Scale, which relates to the patient's excessive thinking about the importance of their pain (Sullivan, Bishop, \& Pivik, 1995). There is a valid Knee Self-Efficacy Scale to evaluate the self-efficacy of the (injured) knee (Thomeé et al., 2006). The ACL-Return to Sport after Injury Scale is used to assess psychological readiness to return to sport by evaluating patient's self-motivation and self-confidence after the RACL. Webster, McPherson, Hewett and Feller (2019) showed that psychological readiness is significantly associated with successful return to sport. The psychological aspect of an athletic identity can also be evaluated by the Athletic Identity Measurement Scale. The results of at least some of the mentioned questionnaires should be more systematically included in rehabilitation protocols and their guidelines/norms for the athlete's optimal return to sport.

The implementation of appropriate psychological interventions into the standard rehabilitation protocols would not only positively affect the return to sport success but could even speed up the process itself and/or improve the functional/physical performance results of an athlete. Rehabilitation programmes that include positive self-talk and goal setting are associated with a greater degree of a patient's compliance than the rehabilitation programmes (Everhart et al., 2015) that can facilitate an athlete's return to sport. Moreover, Cupal and Brewer (2001) found a significant positive effect of guided visualization and relaxation (in addition to the standard rehabilitation) on the knee extensors strength, fear of re-injury and pain level after the RACL. Furthermore, intervention with additional education on surgery and planned recovery can positively influence a rehabilitation process after the RACL. By providing instructional video about the rehabilitation programme to patients before the surgery and in the second and sixth week after the surgery, the researchers were able to significantly reduce patients' perception of the anticipated pain, improve their self-efficacy and even the functionality of the injured knee joint (Maddison, Prapavessis, \& Clatworthy, 2006). That kind of intervention could also help with patient's uncertainty about their rehabilitation progress, which can also have a negative effect (DiSanti et al., 2018). Te Wierike et al. (2013) concluded that psychosocial interventions such as guided therapies (to increase relaxation and self-efficacy), visualizations and additional education of patients about the course of rehabilitation helped to improve the overall rehabilitation outcome after RACL.

In addition to psychological measures, various knee braces or kinesiotaping may help reduce kinesiophobia in individuals after RACL (Harput, Ulusoy, Ozer, Baltaci, \& Richards, 2016). However, caution should be exercised, since these or similar rehabilitation tools/aids can also serve as a constant physical reminder of the injury and thus increase patient's self-awareness of the injured area, which can adversely affect the success of rehabilitation (Burland et al., 2018).

\section{Conclusion}

Our review of the scientific literature revealed a complete lack of research about the possible impact of psychological factors on the success of athlete's rehabilitation and return to sports following musculotendinous 
thigh injuries of the hamstrings, hip adductors or knee extensors. The review additionally revealed that most of the research was done on patients who had RACL. These studies showed several different psychological factors that can significantly affect the outcome of post-injury rehabilitation and an athlete's decision to return to sport. The three most commonly mentioned are fear of injury, self-efficacy, and kinesiophobia.

The same psychological factors could have similar effects in cases of (severe) musculotendinous thigh injuries. Thus, for a more comprehensive and optimal post-injury recovery, it would be reasonable to systematically implement, to the already established rehabilitation protocols, the psychological monitoring of an athlete by using various appropriate questionnaires or interviews. Furthermore, different psychosocial interventions have been shown to improve the athlete's psychological state, which can positively impact the rehabilitation and decision to return to sport. However, before using these tools in clinical practice, methodologically appropriate translation and validation of translations into other languages are required for patients who are not fluent in English.

\section{Acknowledgement}

The study was supported by the Slovenian Research Agency through the project TELASI-PREVENT: Body asymmetries as a risk factor in musculoskeletal injury development: studying aetiological mechanisms and designing corrective interventions for primary and tertiary preventive care (L5-1845).

\section{References}

Ardern, C. L., Taylor, N. F., Feller, J. A., Whitehead, T. S., \& Webster, K. E. (2013). Psychological responses matter in returning to preinjury level of sport after anterior cruciate ligament reconstruction surgery. American Journal of Sports Medicine, 41(7), 1549-1558. doi: 10.1177/0363546513489284

Ardern, C. L., Webster, K. E., Taylor, N. F., \& Feller, J. A. (2011). Return to sport following anterior cruciate ligament reconstruction surgery: A systematic review and meta-analysis of the state of play. British Journal of Sports Medicine, 45(7), 596-606. doi: 10.1136/bjsm.2010.076364

Baez, S. E., Hoch, M. C., \& Hoch, J. M. (2019). Psychological factors are associated with return to preinjury levels of sport and physical activity after ACL reconstruction. Knee Surgery, Sports Traumatology, Arthroscopy. (2019). doi: 10.1007/s00167-019-05696-9

Beijsterveldt, A. M. C. Van, Port, I. G. L. Van De, Vereijken, A. J., \& Backx, F. J. G. (2012). Risk Factors for Hamstring Injuries in Male Soccer Players: 1-10. doi: 10.1111/j.1600-0838.2012.01487.

Booth-Kewley, S., Schmied, E. A., Highfill-McRoy, R. M., Sander, T. C., Blivin, S. J., \& Garland, C. F. (2014). A prospective study of factors affecting recovery from musculoskeletal injuries. Journal of Occupational Rehabilitation, 24(2), 287-296. doi: 10.1007/s10926-013-9456-7

Briet, J. P., Houwert, R. M., Hageman, M. G. J. S., Hietbrink, F., Ring, D. C., \& Verleisdonk, E. J. J. M. (2016). Factors associated with pain intensity and physical limitations after lateral ankle sprains. Injury, 47(11), 2565-2569. doi: 10.1016/j.injury.2016.09.016

Brooks, J. H. M., Fuller, C. W., Kemp, S. P. T., \& Reddin, D. B. (2005). Epidemiology of injuries in English professional rugby union: Part 1 match injuries. British Journal of Sports Medicine, 39(10), 757-766. doi: 10.1136/bjsm.2005.018135

Burland, J. P., Toonstra, J., Werner, J. L., Mattacola, C. G., Howell, D. M., \& Howard, J. S. (2018). Decision to Return to Sport After Anterior Cruciate Ligament Reconstruction, Part I: A Qualitative Investigation of Psychosocial Factors. Journal of Athletic Training, 53(2), 1062-6050-313-316. doi: 10.4085/1062-6050313-16

Cupal, D. D., \& Brewer, B. W. (2001). Effects of relaxation and guided imagery on knee strength, re-injury anxiety, and pain following anterior cruciate ligament reconstruction. Rehabilitation Psychology, 46(1), 28-43. doi: 10.1037/0090-5550.46.1.28

DiSanti, J., Lisee, C., Erickson, K., Bell, D., Shingles, M., \& Kuenze, C. (2018). Perception of rehabilitation and return to sport among high school athletes with anterior cruciate ligament reconstruction: qualitative research study. Journal of Orthopaedic \& Sports Physical Therapy, 48(12), 951-959. doi: 10.1017/ CBO9781107415324.004

Ekstrand, J., Hägglund, M., \& Waldén, M. (2011). Epidemiology of muscle injuries in professional football (soccer). American Journal of Sports Medicine, 39(6), 1226-1232. doi: 10.1177/0363546510395879

Everhart, J. S., Best, T. M., \& Flanigan, D. C. (2015). Psychological predictors of anterior cruciate ligament reconstruction outcomes: a systematic review. Knee Surgery, Sports Traumatology, Arthroscopy, 23(3), 752-762. doi: 10.1007/s00167-013-2699-1

Feeley, B. T., Kennelly, S., Barnes, R. P., Muller, M. S., Kelly, B. T., Rodeo, S. A., \& Warren, R. F. (2008). Epidemiology of National Football League training camp injuries from 1998 to 2007. The American Journal of Sports Medicine, 36(8), 1597-1603. doi: 10.1177/0363546508316021

Grindem, H., Snyder-Mackler, L., Moksnes, H., Engebretsen, L., \& Risberg, M. A. (2016). Simple decision rules can reduce re-injury risk by $84 \%$ after ACL reconstruction: The Delaware-Oslo ACL cohort study. British Journal of Sports Medicine, 50(13), 804-808. doi: 10.1136/bjsports-2016-096031

Harput, G., Ulusoy, B., Ozer, H., Baltaci, G., \& Richards, J. (2016). External supports improve knee performance in anterior cruciate ligament reconstructed individuals with higher kinesiophobia levels. Knee, 23(5), 807-812. doi: 10.1016/j.knee.2016.05.008

Houston, M., Hoch, J., \& Hoch, M. (2017). Collegiate athletes with ankle sprain history exhibit increased fear- 
avoidance beliefs. Journal of sport rehabilitation, 27(5), 419-423.

Houston, M. N., Van Lunen, B. L., \& Hoch, M. C. (2014). Health-related quality of life in individuals with chronic ankle instability. Journal of Athletic Training, 49(6), 758-763. doi: 10.4085/1062-6050-49.3.54

Hsu, C. J., Meierbachtol, A., George, S. Z., \& Chmielewski, T. L. (2017). Fear of re-injury in athletes: implications for rehabilitation. Sports health, 9(2), 162-167.

Kosy, J., Phillips, J., Edordu, A., Pankhania, R., Schranz, P., \& Mandalia, V. (2019). Failure to return to preinjury activity level after hamstring anterior cruciate ligament reconstruction: Factors involved and considerations in goal setting. Indian Journal of Orthopaedics, 53(6), 714-720. doi: 10.4103/ortho. IJOrtho_186_18

Kvist, J., Ek, A., Sporrstedt, K., \& Good, L. (2005). Fear of re-injury: A hindrance for returning to sports after anterior cruciate ligament reconstruction. Knee Surgery, Sports Traumatology, Arthroscopy, 13(5), 393-397. doi: 10.1007/s00167-004-0591-8

Lentz, T. A., Zeppieri, G., George, S. Z., Tillman, S. M., Moser, M. W., Farmer, K. W., \& Chmielewski, T. L. (2015). Comparison of physical impairment, functional, and psychosocial measures based on fear of reinjury/lack of confidence and return-to-sport status after ACL reconstruction. American Journal of Sports Medicine, 43(2), 345-353. doi: 10.1177/0363546514559707

Maddison, R., Prapavessis, H., \& Clatworthy, M. (2006). Modeling and rehabilitation following anterior cruciate ligament reconstruction. Annals of Behavioral Medicine, (17).

Masten, R., Tušak, M., Stražar, K., Zupanc, O., Drobnič, M., Marinšek, M., \& Kandare, M. (2014). Psychological Factors of Rehabilitation of Athletes After Knee Injury. Slovenian Journal of Public Health, 53(3), 226-236.

Miller, R., Kori, S., \& Todd, D. (1991). The Tampa Scale for Kinesiophobia. The Clinical Journal of Pain, 7(1), 51.

Mosler, A. B., Weir, A., Serner, A., Agricola, R., Eirale, C., Farooq, A., ... Crossley, K. M. (2018). Musculoskeletal Screening Tests and Bony Hip Morphology Cannot Identify Male Professional Soccer Players at Risk of Groin Injuries A 2-Year Prospective Cohort Study. The American journal of sports medicine, 46(6), 12941305.

Orchard, J., Seward, H., \& Orchard, J. (2013). Results of 2 decades of injury surveillance and public release of data in the Australian Football League. American Journal of Sports Medicine, 41(4), 734-741. doi: 10.1177/0363546513476270

Sonesson, S., Kvist, J., Ardern, C., Österberg, A., \& Silbernagel, K. G. (2017). Psychological factors are important to return to pre-injury sport activity after anterior cruciate ligament reconstruction: expect and motivate to satisfy. Knee Surgery, Sports Traumatology, Arthroscopy, 25(5), 1375-1384. doi: 10.1007/ s00167-016-4294-8

Sullivan, M. J. L., Bishop, S. R., \& Pivik, J. (1995). The Pain Catastrophizing Scale: Development and validation. Psychological Assessment, 7(4), 524-532. doi: 10.1037/1040-3590.7.4.524

Te Wierike, S. C. M., Van Der Sluis, A., Van Den Akker-Scheek, I., Elferink-Gemser, M. T., \& Visscher, C. (2013). Psychosocial factors influencing the recovery of athletes with anterior cruciate ligament injury: A systematic review. Scandinavian Journal of Medicine and Science in Sports, 23(5), 527-540. doi: 10.1111/ sms. 12010

Thomeé, P., Währborg, P., Börjesson, M., Thomeé, R., Eriksson, B. I., \& Karlsson, J. (2006). A new instrument for measuring self-efficacy in patients with an anterior cruciate ligament injury. Scandinavian Journal of Medicine and Science in Sports, 16(3), 181-187. doi: 10.1111/j.1600-0838.2005.00472.x

Vranceanu, A. M., Bachoura, A., Weening, A., Vrahas, M., Smith, R. M., \& Ring, D. (2014). Psychological factors predict disability and pain intensity after skeletal trauma. Journal of Bone and Joint Surgery - Series A, 96(3), 1-6. doi: 10.2106/JBJS.L.00479

Webster, K. E., McPherson, A. L., Hewett, T. E., \& Feller, J. A. (2019). Factors Associated With a Return to Preinjury Level of Sport Performance After Anterior Cruciate Ligament Reconstruction Surgery. American Journal of Sports Medicine, 47(11), 2557-2562. doi: 10.1177/0363546519865537

Werner, J. L., Burland, J. P., Mattacola, C. G., Toonstra, J., English, R. A., \& Howard, J. S. (2018). Decision to Return to Sport Participation After Anterior Cruciate Ligament Reconstruction, Part II: Self-Reported and Functional Performance Outcomes. Journal of Athletic Training, 53(5), 464-474. doi: 10.4085/10626050-328-16

Wiese-bjornstal, D. M., Smith, A. M., Shaffer, S. M., \& Morrey, M. A. (1998). An integrated model of response to sport injury: Psychological and sociological dynamics. Journal of Applied Sport Psychology, 10(1), 4669. doi: 10.1080/10413209808406377 